\section{Drawing a bead on lymphangiogenesis}

\section{By Tim Fulmer, Senior Writer}

A team of German researchers has developed a high throughput assay to identify compounds that block lymphangiogenesis, a process that promotes the progression and metastasis of solid tumors. ${ }^{1}$ Some of the assay's top hits were in the statin class of drugs, which the researchers now plan to test in preclinical cancer models.

Solid tumors typically metastasize using pre-existing lymphatic vessels, which provide a channel for invasive tumor cells to transit through the body. However, there is growing evidence that metastatic tumors also can induce the formation of new lymphatic vessels, a process known as tumor lymphangiogenesis. ${ }^{2-4}$

In tumor lymphangiogenesis, tumor or stromal cells secrete growth factors that bind receptors on lymphatic cells, triggering the sprouting of new lymphatic vessels at the tumor margin and facilitating metastatic spread of malignant cells to lymph nodes.

However, there were no existing high throughput assays to screen for lymphangiogenesis inhibitors.

Thus, a team of researchers led by Michael Detmar, professor of pharmacogenomics at the Swiss Federal Institute of Technology Zurich (ETHZ), set out to design an automated high throughput assay that mimicked the in situ environment of lymphatic cells and vessels. The resulting assay measures a compound's ability to block lymphatic sprout formation, an early step in the generation of new lymphatic vessels.

To achieve this, the team coated cytodextran beads with human lymphatic endothelial cells (LECs), labeled them with a fluorescent tag and embedded them in a $3 \mathrm{D}$ collagen matrix. The matrix was seeded with three factors known to induce sprouting in the tumor microenvironment: VEGF-A, basic fibroblast growth factor (bFGF) and sphingosine 1-phosphate. The matrix then was placed in a 96-well plate for imaging using fluorescence microscopy.

Cellular protrusions from the bead surface into the surrounding matrix, a proxy for lymphatic sprouting, served as the assay's readout.

The researchers used the assay to screen a commercially available library of 1,280 small molecules with known targets and mechanisms of action.

The screen showed that 31 of the compounds decreased sprouting by at least $20 \%$ compared with vehicle. Of those, about one-third were cytotoxic compounds predicted to kill many cell types and another third were compounds previously identified as angiogenesis and lymphangiogenesis inhibitors.

The final third included compounds that hit a variety of cellular pathways, including U0126, a MEK inhibitor, and mevastatin, an HMGCoA reductase inhibitor.

The mevastatin hit confirmed previous findings from Detmar's lab that showed the compound blocked lymphatic vessel formation in a Xenopus tadpole screen. ${ }^{5}$

The researchers next studied the antilymphangiogenic effects of U0126 and mevastatin in rodents. In mice implanted with a growth factor-secreting gel to induce dermal lymphangiogenesis, both compounds significantly decreased the density of lymphatic vessels in the dermis compared with vehicle $(p<0.001)$.

To confirm that the antilymphangiogenic activity of mevastatin was common to that of statins as a class, the researchers tested three marketed statins: Lipitor atorvastatin from Pfizer Inc., and Mevacor lovastatin and Zocor simvastatin from Merck \& Co. Inc. All three drugs dose-dependently lowered sprouting on the beads at low micromolar concentrations compared with vehicle $(p<0.001)$, with Zocor showing the strongest response.

The researchers next evaluated the inhibitory activity of Zocor in two mouse models of lymphangiogenesis.

In a mouse model of dermal lymphangiogenesis, Zocor significantly decreased dermal lymphatic vessels compared with vehicle $(p<0.001)$. Also, in a mouse model of inflammation-induced corneal lymphangiogenesis, Zocor significantly reduced the number of corneal lymphatic vessels $(p<0.05)$.

Subsequent cell culture studies showed that inhibition of HMG-CoA reductase by Zocor led to downstream loss of the RAC1 protein at the cell membrane and subsequent blockade of sprouting. RAC1 is a GTPase involved in the regulation of cellular processes such as cell growth, cell motility and cytoskeletal organization.

The findings were published in the Proceedings of the National Academy of Sciences.

"One challenge certainly met by this paper is the development of a high throughput assay. That is either difficult or impossible with existing in vivo models of lymphangiogenesis," said Philip Crosier, professor of molecular medicine at The University of Auckland.

A good next step would be to study the hits in a preclinical tumor model, said Crosier.

Readouts from such a study could include "a reduction in the density of peri- and intratumoral lymphatic vessels, a reduction in lymph node occupancy by tumor cells and a reduction in the occurrence of metastatic lesions," he added.

Crosier said he and his Auckland colleagues Jonathan Astin and Kazuhide Okuda have developed transgenic zebrafish lines that could be useful as in vivo screens for compounds that modulate angiogenesis 


\section{ANALYSIS}

and lymphangiogenesis. ${ }^{6,7}$

"We aim to investigate the effects of statins in a range of experimental models with increased lymphangiogenesis, including tumor-induced lymphangiogenesis," corresponding author Detmar told SciBX.

It may also be possible to modify the sprouting assay to screen for inhibitors of cancer invasion by replacing the endothelial coating of the beads with tumor cells, said Detmar.

\section{Getting the right hits}

The sprouting assay in its current form "could have limitations when screening a library of compounds with unknown mechanism of action. For example, the assay might incorrectly identify a nonselective cytotoxic compound as a lymphangiogenesis inhibitor because it appears to reduce sprout formation when in fact it just kills the cells," said André Brändli.

"In the paper, the researchers avoided that problem because they were screening an annotated library of compounds with known targets and mechanisms of action," he said.

Brändli is professor of medicine at the Ludwig Maximilian University of Munich. He was a coauthor with Detmar on a 2009 Blood paper that identified 32 small molecules that interfered with blood vascular or lymphatic development in a Xenopus tadpole screen of compounds targeting angiogenesis and lymphangiogenesis. ${ }^{5}$

Detmar said a next-generation version of the sprouting assay could include an additional automated readout to discriminate cytotoxic agents from lymphangiogenesis inhibitors.

"It may also be important to supplement the sprouting assay with an assay that measures a compound's antiangiogenic activity, to better identify compounds that inhibit lymphangiogenesis without causing toxic inhibition of normal angiogenic processes," said Brändli. “To do that, the researchers might replace the LECs used in the current version of the assay with HUVECs."

Human umbilical vein endothelial cells (HUVECs) are a standard in vitro model system for studying angiogenesis.

"We have adapted the sprouting assay to enable HUVEC-based screens as well," said Detmar. "This will make it possible to screen for specific inhibitors of lymphangiogenesis and/or angiogenesis."

The findings reported in PNAS are not covered by patents, according to Detmar.

Fulmer, T. SciBX 5(37); doi:10.1038/scibx.2012.970

Published online Sept. 20, 2012

\section{REFERENCES}

1. Schulz, M.M.P. et al. Proc. Natl. Acad. Sci. USA; published online Sept. 4, 2012; doi:10.1073/pnas.1206036109

Contact: Michael Detmar, Swiss Federal Institute of Technology Zurich (ETHZ), Zurich, Switzerland e-mail: michael.detmar@pharma.ethz.ch

2. Christiansen, A. \& Detmar, M. Genes Cancer 2, 1146-1158 (2011)

3. Nagahashi, M. et al. World J. Gastroenterol. 16, 4003-4012 (2010)

4. Alitalo, K. Nat. Med. 17, 1371-1380 (2011)

5. Kälin, R.E. et al. Blood 114, 1110-1122 (2009)

6. Okuda, K.S. et al. Development 139, 2381-2391 (2012)

7. Flores, M.V. et al. Dev. Dyn. 239, 2128-2135 (2010)

\section{COMPANIES AND INSTITUTIONS MENTIONED}

Ludwig Maximilian University of Munich, Munich, Germany Merck \& Co. Inc. (NYSE:MRK), Whitehouse Station, N.J. Pfizer Inc. (NYSE:PFE), New York, N.Y.

Swiss Federal Institute of Technology Zurich, Zurich, Switzerland The University of Auckland, Auckland, New Zealand 